\title{
UM RELATO DE EXPERIÊNCIA ACERCA DAS POTENCIALIDADES E DESAFIOS DA RESIDÊNCIA MULTIPROFISSIONAL EM PEDIATRIA: SOB A ÓTICA DA ENFERMAGEM
}

\author{
AN EXPERIENCE REPORT ABOUT THE POTENTIALS AND CHALLENGES OF \\ MULTIDISCIPLINARY RESIDENCY IN PEDIATRICS: FROM THE PERSPECTIVE OF \\ NURSING
}

\author{
Keyze Mirelly Carneiro da Silva Ferreira ${ }^{1}$ \\ Neirice Rodrigues Alves de Vasconcelos ${ }^{2}$
}

RESUMO: O presente relato de experiência expõe as vivências e os desafios de enfermeiras inseridas em um programa de residência integrada multiprofissional em saúde hospitalar com ênfase em saúde da criança e do adolescente do estado da Paraíba. Definida como como modalidade de pós-graduação lato sensu, a residência foi realizada através do ensino em serviço em um hospital universitário e exigiu das residentes o cumprimento das 6o horas semanais em regime de dedicação exclusiva, totalizando 5.760 horas entre $80 \%$ de atividades práticas e $20 \%$ de atividades teóricas distribuídas em dois anos de serviço. Em teoria, no primeiro ano os residentes atuariam apenas no hospital promotor do programa, conhecendo o processo de trabalho de diferentes setores, desde a clínica pediátrica até unidades de terapias intensivas. No segundo ano, intitulado $\mathrm{R}_{2}$, o ensino em serviço se daria por cenários externos ao referido hospital, a fim de conhecer os pontos de atenção da rede de atenção à saúde, com foco em saúde da criança e do adolescente. Entretanto, devido à pandemia da COVID-ı, o calendário institucional sofreu modificações devido à necessidade de adaptação ao novo cenário pandêmico. Ainda assim, a carga horária exaustiva de 60 horas semanais, rotina e a alta demanda de atividades promoveram cansaço físico e mental, trazendo consequências emocionais e psicológicas. Em contrapartida, proporcionou a aquisição de novas experiências, conhecer novas rotinas, novas realidades e trocar conhecimentos, potencializando as habilidades das enfermeiras residentes na sua área de atuação, preparando-as para desafios futuros a serem vivenciados no mercado de trabalho.

Palavras-chaves: Enfermagem. Residência Hospitalar. Educação de Pós-Graduação em Enfermagem.

ABSTRACT: This experience report exposes the experiences and challenges of nurses inserted in a multiprofessional integrated residency program in hospital health with an emphasis on child and adolescent health in the state of Paraíba. Defined as a lato sensu postgraduate modality, the residency was carried out through in-service teaching at a

\footnotetext{
${ }^{1}$ Graduação em Enfermagem pelo Centro Universitário de João Pessoa (UNIPÊ). Residente em Saúde Hospitalar com ênfase em Saúde da Criança e do Adolescente pelo Programa Integrado de Residência em Saúde Hospitalar (RIMUSH) - HULW/UFPB. keyzemirellyı23@gmail.com

${ }^{2}$ Graduação em Enfermagem pela Universidade Estadual Vale do Acaraú (UVA). Residente em Saúde Hospitalar com ênfase em Saúde da Criança e do Adolescente pelo Programa Integrado de Residência em Saúde Hospitalar (RIMUSH) - HULW/UFPB. neirice.1@gmail.com
} 
university hospital and required residents to comply with 60 hours per week in exclusive dedication regime, totaling 5,760 hours among $80 \%$ of practical activities and $20 \%$ of theoretical activities spread over two years of service. In theory, in the first year, residents would only work at the hospital that sponsors the program, knowing the work process in different sectors, from the pediatric clinic to intensive care units. In the second year, entitled $\mathrm{R}_{2}$, in-service teaching would take place in scenarios outside the aforementioned hospital, in order to know the points of attention of the health care network, with a focus on child and adolescent health. However, due to the COVID-I9 pandemic, the institutional calendar was modified due to the need to adapt to the new pandemic scenario. Even so, the exhaustive workload of 6o hours per week, routine and the high demand for activities promoted physical and mental fatigue, bringing emotional and psychological consequences. On the other hand, it provided the acquisition of new experiences, knowing new routines, new realities and exchanging knowledge, enhancing the skills of nurses residing in their area of expertise, preparing them for future challenges to be experienced in the labor market.

Keywords: Nursing. Hospital Residence. Graduate Nursing Education.

\section{INTRODUÇÃO}

As residências multiprofissionais e em áreas profissionais da saúde foram instituídas através da promulgação da lei no 11.129 de 2005, sendo definidas como modalidades de pós-graduação lato sensu, contemplando as profissões da área da saúde, exceto os médicos (BRASIL, 2005).

Voltados para a educação em serviço, os programas de residências multiprofissionais contemplam os profissionais de enfermagem, fisioterapia, bioquímica, ciências biológicas, educação física, farmácia, fonoaudiologia, medicina veterinária, nutrição, odontologia, terapia ocupacional, psicologia, serviço social, saúde coletiva e física médica. Além da interação interdisciplinar, essa modalidade de especialização almeja a inserção de profissionais qualificados no mercado de trabalho (BRASIL, 202I).

No mais, exige-se que os programas de residências devem ser organizados segundo às lógicas organizacional da Rede de Atenção a Saúde (RAS) e da gestão do Sistema Único de Saúde (SUS), considerando as peculiaridades loco-regionais e especificidades de cada núcleo profissional contemplado (BRASIL, 2012).

Fruto da parceria entre o setor saúde e educação, a residência em saúde tem carga horária de 6o horas semanais e duração mínima de dois anos de dedicação exclusiva, sendo o residente impossibilitado de trabalhar ou cursar outros tipos de graduação/pós-graduação durante o ensino em serviço (BRASIL, 202I). 
Os programas de residências devem contemplar atividades práticas, teóricas e prático-teóricas. No tocante às atividades teóricas, o tutor tem um papel imprescindível na orientação acadêmica de orientar os preceptores e os residentes, instituindo tutorias individuais por núcleos profissionais e tutorias de campo (BRASIL, 2012).

O preceptor, por sua vez, supervisiona o residente nas atividades práticas nos serviços de saúde, devendo orientá-lo, acompanhá-lo, fornecer suporte e integrá-lo com a equipe de saúde (BRASIL, 2012).

A Residência Multiprofissional em Saúde Hospitalar com ênfase em Saúde da Criança e do Adolescente, definida como modalidade de pós-graduação lato sensu, realizada através do ensino em serviço em um Hospital Universitário (HU) na Paraíba, exige dos residentes o cumprimento das 6o horas semanais em regime de dedicação exclusiva, totalizando 5.760 horas entre $80 \%$ de atividades práticas e $20 \%$ de atividades teóricas distribuídas em dois anos de serviço.

Conforme a Universidade Federal de Juiz de Fora (202I), o trabalho do tipo relato de experiência contextualiza, de forma objetiva e baseado na literatura, as experiências diante das dificuldades, das vivências profissionais e das reflexões, contribuindo para a formação de espaços de discussões em prol da melhoria do cuidado na saúde.

A partir desses direcionamentos, relata-se as experiências de duas residentes de enfermagem do programa de saúde hospitalar com ênfase em saúde da criança e do adolescente durante os dois anos de ensino em serviço, quanto aos caminhos traçados e desafios encontrados, a fim de instigar a melhoria contínua dos processos de formações dos residentes em saúde.

\section{RELATO DE EXPERIÊNCIA}

Trata-se de um estudo descritivo, do tipo relato de experiência, elaborado a partir das vivências de duas enfermeiras residentes em saúde hospitalar com ênfase em saúde da criança e do adolescente, participantes do programa de residência integrada multiprofissional em saúde hospitalar vinculado a um hospital universitário do estado da Paraíba.

O programa de residência supracitado iniciou suas atividades no início do mês de março de 2020 e, conforme as legislações vigentes supracitadas, é pautada no ensino em 

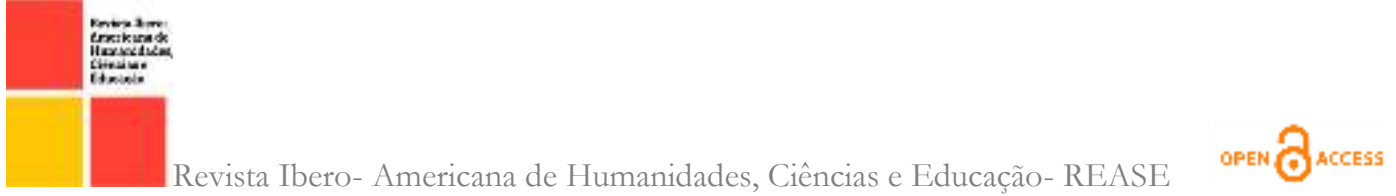

serviço com duração de dois anos, sendo 6o horas semanais e totalizando 5.760 horas de atividades práticas e teórico-práticas, em regime de dedicação exclusiva.

A ênfase em Saúde da Criança e do Adolescente disponibilizou ro vagas conquistadas mediante concurso público, sendo constituídas por 02 vagas de enfermagem, 02 de fisioterapia, or de farmácia, or de terapia ocupacional, or de fonoaudiologia, or de psicologia, or de serviço social e or de nutrição.

Ademais, a parceria firmada entre o programa e o hospital universitário possibilitou a construção de espaços de trocas de conhecimentos e experiências, viabilizando os cenários de prática e o trabalho em equipe na perspectiva da integralidade e interdisciplinaridade.

Em teoria, no primeiro ano os residentes atuariam apenas no hospital promotor do programa, conhecendo o processo de trabalho de diferentes setores, desde a clínica pediátrica até unidades de terapias intensivas. No segundo ano, intitulado R2, o ensino em serviço se daria por cenários externos ao referido hospital, a fim de conhecer os pontos de atenção da rede de atenção à saúde, com foco em saúde da criança e do adolescente.

Entretanto, devido à pandemia da COVID-ı9, o calendário institucional sofreu modificações devido à necessidade de adaptação ao novo cenário pandêmico, estendendo a atuação dos residentes no HU por um período maior do que o planejado, totalizando cerca de um ano e três meses.

A carga horária de 60 horas semanais foi fracionada em $80 \%$ de atividades práticas e 20\% de atividades teóricas e prático-teóricas, ou seja, cerca de 48 e 18 horas respectivamente. Cada residente deveria seguir o calendário do programa, havendo turnos reservados para tutorias de núcleo e de campo, além de reuniões.

No primeiro ano de residência, devido à pandemia do novo coronavírus, as tutorias de enfermagem foram online, sendo realizadas através da participação em cursos da área profissional realizados pelo próprio tutor ou por terceiros. Ademais, a tutoria de núcleo contempla as três ênfases da residência: saúde da criança e do adolescente, saúde do idoso e paciente crítico.

À vista disso, durante a vivência na prática, foram elaborados alguns casos clínicos de acordo com cada campo de atuação, sendo discutidos em encontros pontuais pertinentes às atividades de tutoria. 

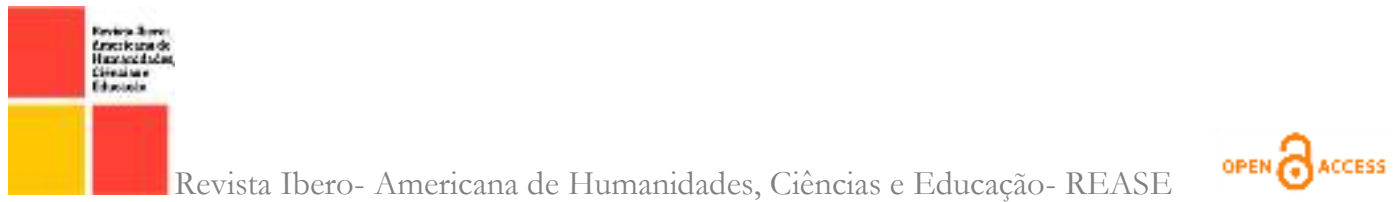

No mais, para compor a carga horária teórica, fez-se necessários encontros de ênfase, ou seja, entre os diferentes níveis profissionais que constituíam a equipe multiprofissional de residentes em saúde da criança e do adolescente. Nessas reuniões, discutiam-se casos clínicos, desafios e potencialidades em relação à vivência de cada núcleo.

As residentes atuaram, no primeiro ano, na clínica médica pediátrica, na Unidade de Cuidados Intermediário Neonatal (UCIN), na Unidade de Cuidados Intermediários Canguru (UCINCA), na Unidade de Terapia Intensiva Neonatal (UTIN) e Unidade de Terapia Intensiva (UTI) pediátrica.

Os profissionais de enfermagem do referido hospital universitário possuíam capacidade técnica e científica para atuarem como preceptores das residentes. Sempre se demonstraram atenciosos e preocupados com o aprendizado e procuraram contribuir de forma significativa na formação das enfermeiras.

Foi um ano de vivências valiosas que contribuíram para a formação profissional do enfermeiro na área de saúde da criança e do adolescente, ademais, proporcionou interação e troca de conhecimentos e experiências com as diversas equipes dos setores.

No decorrer do ano, várias atividades multiprofissionais, com foco na humanização da assistência, foram efetuadas pela equipe de residente. Datas importantes como o dia das crianças e o halloween foram comemoradas na clínica pediátrica juntamente com as crianças internadas, obedecendo as devidas regras de distanciamento social.

Na UTIN e na UCIN, elaborou-se e implementou-se o projeto do mêsversário, planejado a priori pela terapeuta ocupacional da equipe e introduzido pela equipe. Outrossim, a data comemorativa "São João" foi celebrada juntamente com a equipe e os pequenos internos na UTI Pediátrica.

As enfermeiras residentes sempre eram responsáveis por cuidar dos menores e adiantar os procedimentos de rotina para que houvesse as comemorações, com a finalidade de trazer um pouco de alegria, amenizando os desafios promovidos pela internação.

No segundo ano, com mais conhecimento e experiência, as residentes dirigiram-se aos cenários externos como complementação do processo de formação profissional. A priori, o primeiro cenário de prática foi um hospital referência em oncologia na Paraíba. 

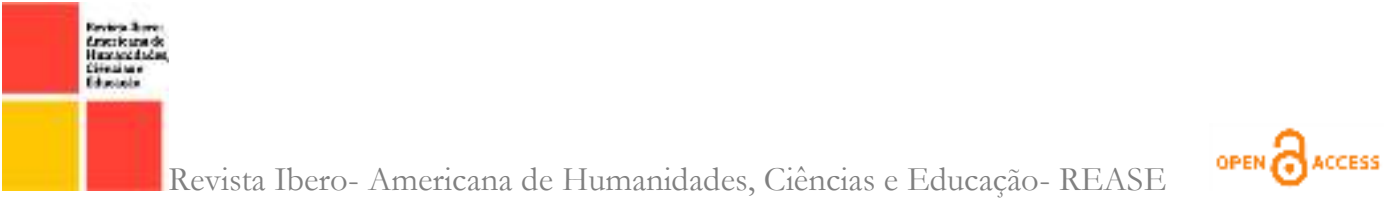

Nessa instituição, a enfermagem atuou na clínica pediátrica, no ambulatório de oncologia pediátrico e na UTI pediátrica.

O segundo cenário do último ano foi um complexo hospitalar referência em pediatria no estado, no qual as residentes tiveram oportunidade de conhecer e trabalhar na clínica médica e cirúrgica pediátrica e UTI pediátrica.

O terceiro cenário contemplou uma maternidade municipal da Paraíba, referência na Iniciativa Hospital Amigo da Criança (IHAC), local onde as enfermeiras residentes atuaram por três meses na Admissão de Recém-Nascido, UCINCA, UCIN e UTIN.

O segundo e último ano foi rico em aperfeiçoamento das práticas e conhecimentos das residentes pelo fato de conhecer as diferentes realidades e instituições de referência no Estado. Em contrapartida, o núcleo de enfermagem apresentou algumas limitações em relação à tutoria devido aos desencontros entre o tutor e os residentes, fragilizando os encontros teóricos relacionados à tutoria de núcleo.

Em relação aos desafios, destaca-se a carga horária exaustiva de 6o horas semanais. A rotina e a alta demanda de atividades promoveram cansaço físico e mental, trazendo consequências emocionais e psicológicas. Ademais, foi comum a sensação de pressão imposta indiretamente pelos profissionais dos serviços sobre as residentes, havendo a necessidade de se sentir útil e demonstrar proatividade durante todo o plantão.

Outro impasse percebido foi a integração com a equipe médica e residentes médicos, que constitui, a depender do serviço, um grande ponto de fragilidade, dificultando a comunicação efetiva e envolvimento nas práticas exercidas.

Paralelo a tudo isso, a residência multiprofissional em saúde da criança e do adolescente proporcionou a aquisição de novas experiências, conhecer novas rotinas, novas realidades e trocar conhecimentos com as equipes através da modalidade ensino em serviço, potencializando as habilidades das enfermeiras residentes na sua área de atuação.

\section{CONSIDERAÇÕES FINAIS}

Com base no relato exposto sobre a atuação das enfermeiras residentes em saúde hospitalar com ênfase em saúde da criança e do adolescente nos diferentes setores e serviços paraibanos, percebe-se que a modalidade ensino-serviço facilitou o processo de aprendizagem e aquisição de experiências e habilidades. 
Ademais, trabalhar em equipe multiprofissional possibilitou o compartilhamento de saberes e a troca conhecimentos, ampliando a ótica de cada profissional em prol de uma melhor qualidade da assistência prestada aos menores.

Por conseguinte, foi importante perceber que a intensidade da carga horária, a diversidade dos cenários de atuação e alta demanda de atividades preparam o profissional residente para os desafios futuros a serem vivenciados no mercado de trabalho.

A utopia está lá no horizonte. Me aproximo dois passos, ela se afasta dois passos. Caminho dez passos e o horizonte corre dez passos. Por mais que eu caminhe, jamais alcançarei. Para que serve a utopia? Serve para isso: para que eu não deixe de caminhar" (Fernando Birri).

Pensar que a residência em enfermagem será perfeita é uma utopia, mas deve-se caminhar nessa direção em busca da melhoria contínua das condições de estrutura, ensino em serviço e de trabalho dignas e adequadas ao desempenho das atribuições.

\section{CONTRIBUIÇÃO DOS AUTORES}

KMCSF e NRAV realizaram a revisão da literatura e relataram suas experiências descrita no corpo do trabalho. As autoras revisaram o manuscrito final e aprovaram a sua submissão.

\section{REFERÊNCIAS BIBLIOGRÁFICAS}

BRASIL. Lei no 11.129 , de 30 de junho de 2005. Institui o Programa Nacional de Inclusão de Jovens. Diário Oficial da União. Brasília, DF, jan. 2005. Disponível em: http://www.planalto.gov.br/ccivil_03/_ato2004-2006/2005/lei/lin29.htm. Acesso em: I3 dez. 202I.

BRASIL. Resolução no 2, de 13 de abril de 2012. Ministério da Educação. Secretaria de Educação Superior. Comissão Nacional de Residência Multiprofissional em Saúde. Diário Oficial da União. Brasília, DF, abr. 2012. Disponível em: https://abmes.org.br/arquivos/legislacoes/Res-CNRM-oo2-2012-04-13.pdf. Acesso em: is dez. 202I.

BRASIL. Portaria Interministerial $\mathrm{n}$ o 7 , de $\mathrm{i} 6$ de setembro de 2021. Dispõe sobre a estrutura, a organização e o funcionamento da Comissão Nacional de Residência Multiprofissional em Saúde. Diário Oficial da União. Brasília, DF, set. 202r. Disponível em: https://www.in.gov.br/en/web/dou/-/portaria-interministerial-n-7-de-16-desetembro-de-202I-345462405. Acesso em: 13 dez. 2021.

Universidade Federal de Juiz de Fora. Instituto de Ciências da Vida. Departamento de Nutrição. INSTRUTIVO PARA ELABORAÇÃO DE RELATO DE EXPERIÊNCIA. Estágio em Nutrição em Saúde Coletiva. Disponível em: 
https://www.ufjf.br/nutricaogv/files/2016/o3/Orienta\%c3\%a7\%c3\%bses-

Elabora\%c3\%a7\%c3\%a3o-de-Relato-de-Experi\%c3\%aancia.pdf. Acesso em: 13 dez. 2021. 